\title{
Genome-wide identification, evolution, and expression of the SNARE gene family in wheat resistance to powdery mildew
}

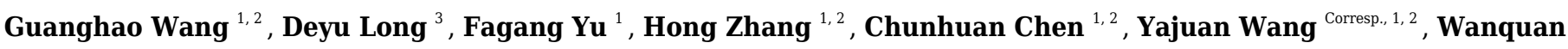 \\ Ji Corresp. 1,2 \\ ${ }^{1}$ College of Agronomy, Northwest A\&F University, Yangling, No.3 Taicheng Road, China \\ 2 Shaanxi Research Station of Crop Gene Resources and Germplasm Enhancement, Ministry of Agriculture, Yangling, Shaanxi, China \\ 3 College of Life Sciences, Northwest A\&F University, Yangling, Shaanxi, No.3 Taicheng Road, China \\ Corresponding Authors: Yajuan Wang, Wanquan Ji \\ Email address: wangyj7604@163.com, jiwanquan2008@126.com
}

SNARE proteins mediate eukaryotic cell membrane/transport vesicle fusion and act in plant resistance to fungi. Herein, 173 SNARE proteins were identified in wheat and divided into 5 subfamilies and 21 classes. The number of the SYP1 class type was largest in TaSNAREs. Phylogenetic tree analysis revealed that most of the SNAREs were distributed in 21 classes. Analysis of the genetic structure revealed large differences among the 21 classes, and the structures in the same group were similar, except across individual genes. Excluding the first homoeologous group, the number in the other homoeologous groups was similar. The 2000 bp promoter region of the TASNARE genes were analyzed, and many W-box, MYB and disease-related cis-acting elements were identified. The qRT-PCR-based analysis of the SNARE genes revealed similar expression patterns of the same subfamily in one wheat variety. The expression patterns of the same gene in resistant/sensitive varieties largely differed at $6 \mathrm{~h}$ after infection, suggesting that SNARE proteins play an important role in early pathogen infection. Here, the identification and expression analysis of SNARE proteins provide a theoretical basis for studies of SNARE protein function and wheat resistance to powdery mildew. 
1 Genome-wide identification, evolution, and expression of the

\title{
$S N A R E$ gene family in wheat resistance to powdery mildew
}

\author{
Guanghao Wang ${ }^{1,2}$, Deyu Long ${ }^{3}$, Fagang $\mathrm{Yu}^{1,2}$, Hong Zhang ${ }^{1,2}$, Chunhuan $\mathrm{Chen}^{1,2}$, Yajuan Wang ${ }^{1,2} \&$ \\ Wanquan $\mathrm{Ji}^{1,2}$ \\ 1 College of Agronomy, Northwest A\&F University, Yangling 712100, Shaanxi, China \\ 2 Shaanxi Research Station of Crop Gene Resources and Germplasm Enhancement, Ministry of \\ Agriculture, Yangling 712100, China \\ 3 College of Life Sciences, Northwest A\&F University, Yangling, Shaanxi, China \\ Correspondence and requests for materials should be addressed to Yajuan Wang (wangyj7604@163.com) \\ and Wanjuan Ji (jiwanquan2008@126.com).
}

\begin{abstract}
SNARE proteins mediate eukaryotic cell membrane/transport vesicle fusion and act in plant resistance to fungi. Herein, 173 SNARE proteins were identified in wheat and divided into 5 subfamilies and 21 classes. The number of the SYP1 class type was largest in TaSNAREs. Phylogenetic tree analysis revealed that most of the SNAREs were distributed in 21 classes. Analysis of the genetic structure revealed large differences among the 21 classes, and the structures in the same group were similar, except across individual genes. Excluding the first homoeologous group, the number in the other homoeologous groups was similar. The 2000 bp promoter region of the TaSNARE genes were analyzed, and many Wbox, MYB and disease-related cis-acting elements were identified. The qRT-PCR-based analysis of the SNARE genes revealed similar expression patterns of the same subfamily in one wheat variety. The expression patterns of the same gene in resistant/sensitive varieties largely differed at $6 \mathrm{~h}$ after infection, suggesting that SNARE proteins play an important role in early pathogen infection. Here, the identification and expression analysis of SNARE proteins provide a theoretical basis for studies of SNARE protein function and wheat resistance to powdery mildew.
\end{abstract}

Key words: Wheat; SNARE; Genome-wide; Expression pattern; Gene family

\section{INTRODUCTION}

SNARE (soluble N-ethylmaleimide sensitive factor attachment protein receptor) proteins are employed for a significant number of vital transport processes, as they mediate the fusion of the membranes of cargo-containing small shuttles, which are referred to as vesicles, and target membranes(Lipka et al. 2007). These proteins are involved in vesicle membrane fusion and are 
32

33

34

35

36

37

38

39

40

41

42

43

44

45

46

47

48

49

50

51

52

53

54

55

56

57

58

59

60

61

62

63

64

65

66

67

68

69

70

71

72

responsible for transport in the endomembrane system, as well as for endocytosis and exocytosis. According to their functions, SNARE proteins can be divided into vesicle-associated (v-SNARE) and target-membrane-associated (t-SNARE) proteins (Söllner et al. 1993). Alternatively, SNAREs can be grouped as Q-SNAREs and R-SNAREs. These proteins have either conserved glutamine or conserved arginine residues in the center of the SNARE domain, and Q-SNAREs can be further subdivided into Qa-SNAREs, Qb-SNAREs, and Qc-SNAREs (Bock et al. 2001). SNAP-25-like proteins comprise Qb-SNARE and Qc-SNARE motifs (Schilde et al. 2008). RSNAREs have an either short or long N-terminal regulatory region, further subdividing them into brevins and longins (Lipka et al. 2007). Previous studies have shown the existence of 60 SNARE proteins in Arabidopsis thaliana, 57 SNAREs in Oryza sativa, 69 SNAREs in Populus trichocarpa (Lipka et al. 2007), 27 SNAREs in wheat (Gaggar et al. 2020) and 21 syntaxins in Solanum lycopersicum (Bracuto et al. 2017). In addition, Sanderfoot revealed the evolution of eukaryotic SNAREs (Sanderfoot 2007).

The plant cell endomembrane secretion pathway plays an important role in the interaction between plant cells and microbes (Snyder \& Nicholson 1990; Walther-Larsen et al. 1993). Plant cells are capable of identifying pathogen-associated molecular patterns through surface receptors, and cell surface receptor proteins recognize signal peptides. It was shown that the processing and positioning of these receptors occur through the protein secretion pathway (Wang \& Dong 2011). The autoimmunity of plants to infiltration by powdery mildew fungi is accomplished by targeting the cell wall with papillary factors, including purines, cytoplasmic components, extracellular membrane components and SYP121/PEN1 (Nielsen et al. 2012). In Arabidopsis, PEN1 (SYP121) and its closest homolog, SYP122, appear to have a fundamental function in secretion and specific defense-related functions at the plant cell wall (Assaad et al. 2004; Collins et al. 2003). Similarly, HvROR2 (Collins et al. 2003) and SiPEN1 (Bracuto et al. 2017) were shown to be associated with defense against powdery mildew fungi. The AtSYP121/AtPEN1AtSNAP33-AtVAMP-721/722 protein complex can assist cell emesis at sites of fungal invasion (Douchkov et al. 2005; Kwon et al. 2008; Lipka et al. 2008; Wick et al. 2003). In addition, AtSEC11 modulates PEN1-dependent vesicle trafficking by dynamically competing for PEN1 binding with VAMP721 and SNAP33 (Karnik et al. 2013).

MdSYP121 affects the pathogen infection process in apples by regulating the salicylic acid (SA) pathway and the oxidation-reduction process (He et al. 2018). The SYP4 group regulates both secretory and vacuolar transport pathways and the related extra cellular resistance to fungal pathogens (Uemura et al. 2012). NbSYP132 may act as a cognate target SNARE protein receptor and positively regulate the exocytosis of vesicles containing antibacterial pathogenesis-related (PR) proteins (Kalde et al. 2007). Silencing of StSYRl enhances the resistance of potato to Phytophthora infestans (Eschen-Lippold et al. 2012).

OsVAMP714 can regulate disease resistance to blast in rice, but OsVAMP7111 cannot. Furthermore, OsVAMP714 overexpression promotes leaf sheath elongation (Sugano et al. 2016). Ectopic expression of AtBET12 had no inhibitory effect on general ER-Golgi anterograde transport but led to intracellular accumulation of PR1 (Chung et al. 2018). GOS12 was an essential host factor for plasmodesmata (PD) targeting of the P3N-PIPO protein to defend 
against soybean mosaic virus (Song et al., 2016). AtMEMB12 was targeted by miR393b* to modulate the exocytosis of antimicrobial PR1 (Zhang et al. 2011). AtSyp71 was a host factor that was essential for successful viral infection, mediating the fusion of virus-induced vesicles with chloroplasts during TuMV infection (Karnik et al. 2013). OsSEC3A enhances rice resistance to Magnaporthe oryzae by negatively regulating the pathogenesis and expression of SA synthesis-related genes (Ma et al. 2018). TaNPSN11, TaNPSN13, and TaSYP132 have diverse functions in the prevention of Pst infection and hyphal elongation (Wang et al. 2014).

Wheat is an important food crop in the world, but its output is subject to various severe biological and abiotic stresses. Wheat powdery mildew may occur in each growth period of wheat, which is mainly manifested in the leaves and cases. The main symptom is the appearance of a white powdery mildew layer on the leaf surface, which gradually expands and unites to constitute an oval mold and an irregular mold layer, as well as a layer of powdery substance (conidia) on the surface. In severe cases, a gray mold layer will be established on the leaves and black particles will appear. After the stems and leaves are infected, the wheat was prone to lodging and shrinking without heading. Discovering and using resistance genes is an environmentally friendly and economical way to resist wheat powdery mildew. Herein, 173 SNARE proteins were identified in wheat. Phylogenetic tree analysis revealed that most of the SNAREs were distributed in 21 classes. TaSNARE genes include many W-box, MYB and disease-related cis-elements in the promotor region. The expression patterns largely differed at $6 \mathrm{~h}$ after infection with powdery mildew. This study aimed to develop a better understanding of the identification, evolution, and expression of SNARES and explore the relationship between wheat SNAREs and powdery mildew.

\section{Materials and Methods}

\subsection{Identification of TaSNARE genes}

The wheat genomes and annotations used were from the newest IWGSC (International Wheat Genome Sequencing Consortium) v1.0 (https:/wheat-urgi.versailles.inra.fr/SeqRepository). The hidden Markov models (HMMs) of the SNARE (PF05739), Syntaxin (PF00804), longin (PF13774), Synaptobrevin (PF00957), SEC20 (PF03908), V-SNARE-C (PF12352), V-SNARE (PF05008) and USE1 (PF09753) motifs were downloaded from the Pfam database (http://pfam.sanger.ac.uk/). The wheat SNARE protein sequences were analyzed with HMMER 3.0 (http://hmmer.janelia.org/) as the query and the default parameters $(\mathrm{E}<0.01)$. All presumptive $S N A R E$ genes were retained and confirmed using the Pfam database and the NCBI conserved domain database (https://www.ncbi.nlm.nih.gov/Structure/cdd/wrpsb.cgi). The molecular weights (MW) and protein isoelectric point (pI) of the TaSNARE genes were obtained using the tools from the ExPASy website (https://web.expas y.org/compute_pi/).

Multiple alignments of SNARE proteins were performed using ClustalW (Larkin et al. 2007) in MEGA 7.0 (http://www.megasoftware.net/). Phylogenetic analyses were performed using the 
110 NJ (neighbor-joining) method in MEGA 7.0 (Kumar et al. 2016) with 1000 bootstrap resampling, 111 the Jones-Taylor-Thornton (JJT) model (Jones et al. 1992), and the pairwise deletion option. 112 Gene Ontology (GO) enrichment analysis of SNAREs was implemented using the clusterProfier R 113 package $(\mathrm{R}$ version=4.0.3).

\subsection{Exon/intron structure analysis, conserved motif identification and cis-acting elements} analysis

The gene structure provides important information, including disaggregated and evolutionary relationships among gene families. The SNARE genomic sequences and CDS sequences extracted from the plant database were compared in the gene structure display server program to determine the exon/intron organization of $S N A R E$ genes. The default parameters were used in the Multiple Em for Motif Elicitation (MEME) (http://meme-suite.org/) program for the identification of conserved protein motifs and a maximum number of 15 motifs. Promoter of the SNARE gene was used to analyze the cis-acting elements as previously described by Sun et al., 2017.

\subsection{Chromosomal locations and gene collinearity analysis}

The physical chromosome locations of all SNAREs were obtained from the gff3 files of wheat databases. TBtools (https:/github.com/CJ-Chen/TBtools) software was adopted to visually map the chromosomal location. Gene duplication events were analyzed using the Multiple Collinearity Scan toolkit (MCScanX: http://chibba.pgml.uga.edu/mcscan2/). To exhibit segmentally duplicated pairs and orthologous pairs of SNARE genes, we used Dual Systeny Plotter software (https://github.com/CJ-Chen/TBtools) to drawn collinearity maps.

\subsection{Fungus and Wheat materials}

The wheat-Ae. geniculata disomic addition line NA0973-5-4-1-2-9-1 (CS-SY159 DA 7M, (CS)/Ae. geniculata SY159//CS)) was used (Wang et al. 2016). 'Shaanyou 225' was the powdery mildew susceptible control variety. The wheat-Ae. geniculata disomic addition line TA7661 (CS-AEGEN DA 7M) was kindly provided by Dr. Friebe BR and Dr. Jon Raupp of the Department of Plant Pathology, Throckmorton Plant Sciences Center, Kansas State University, Manhattan, KS, USA. The powdery mildew isolates E09 was maintained on the susceptible wheat 'Shaanyou 225'. All plants were cultured in a growth chamber with soil at $18^{\circ} \mathrm{C}$ under a $16 \mathrm{~h}$ light/8h dark photoperiod. The 14-day-old seedlings were inoculated with powdery mildew conidia from 'Shaanyou 225' seedlings infected 10 days previously using the dusting method. Wheat leaves were collected at $0 \mathrm{~h}, 6 \mathrm{~h}, 12 \mathrm{~h}, 24 \mathrm{~h}$, and $48 \mathrm{~h}$ after powdery mildew infection, and quickly put into cryopreservation tubes and stored in liquid nitrogen. The leaves were used for 
143 the next step of RNA extraction and q-PCR experiments. The method by Wang et al., 2020 was

144

145

146

147

148

149

150

151

152

153

154

155

156

157

158

159

160

161

162

163

164

165

166

167

168

169

170

171

172

173

174

175

176

177

178

used for CS (Chinese Spring), 7M CH (NA0973-5-4-1-2-9-1), 7M US (TA7661) and 'Shaanyou $225^{\prime}$ to identify powdery mildew.

\subsection{RNA-seq expression analysis of SNARE genes}

To further understand the function of the SNARE gene, we investigated the reported RNAseq data, including the developmental timecourse in five tissues (Choulet et al. 2014), grain layers (Pearce et al. 2015), grain layer developmental timecourse (Pfeifer et al. 2014), senescing leaves timecourse (Pearce et al. 2014), photomorphogenesis of DV92 and G3116 (Fox et al. 2014), and drought and heat effects (Liu et al. 2015). The data were analyzed using MeV (Multi Experiment Viewer) software. Data obtained from the RNA-seq expression atlas were normalized based on the mean expression value of each gene in all tissues/organs analyzed and clustered by the hierarchical clustering method.

The developmental time course in five tissues includes all of the wheat stage, as follows (Zadoks et al. 1974): seeding (first leaf through coleoptile, Zadoks Scale 10, Z10), three leaves (3 leaves unfolded, Z13), three tillers (Main shoot and 3 tillers, Z23), spike at $1 \mathrm{~cm}$ (pseudostem erection, Z30), two nodes(2nd detectable node, Z32), meiosis (flag leaf ligule and collar visible, Z39), anthesis (1/2 of flowering complete, Z65), 2 days after anthesis (DAA) (Kernel (caryopsis) watery ripe, Z71), 14 DAA(medium Milk, Z75), and 30 DAA (soft dough, Z85). The grain layers contained three parts at 12 days post-anthesis (DPA): the outer pericarp, inner pericarp, and endosperm. The grain layer developmental timecourse included the following seven-stages: 10 DPA whole endosperm, 20 DPA whole endosperm, 20 DPA starchy endosperm, 20 DPA transfer cells, 20 DPA aleurone, 30 DPA starchy endosperm, and 30 DPA aleurone plus endosperm. The senescing leaves timecourse contains three stages: heading date (HD), 12 DAA and 22 DAA. The photomorphogenesis of the wild winter wheat $T$. monococcum ssp. aegilopoides (accession G3116) and the domesticated spring wheat $T$. monococcum ssp. monococcum (accession DV92) was investigated. Drought and heat effect examinations included seven treatments, as follows: control, drought $1 \mathrm{~h}$, drought $6 \mathrm{~h}$, heat $1 \mathrm{~h}$, heat $6 \mathrm{~h}$, and drought plus heat $1 \mathrm{~h}$, drought plus heat $6 \mathrm{~h}$. Powdery mildew pathogen stress: included non-innoculation, powdery $24 \mathrm{~h}$, powdery $48 \mathrm{~h}$ and powdery $72 \mathrm{~h}$.

\subsection{RNA extraction and real-time quantitative PCR}

The total RNA was extracted from samples of fungal inoculated leaves using the optimized extraction procedure described by Zhang et al., 2014.

The SYBR Green Premix Ex Taq ${ }^{\text {TM }}$ II quantitative PCR system (Takara, Dalian) was used for qPCR analysis. All experiments involving q-PCR were performed on a Q7 Real-Time PCR System (Applied Biosystems, Foster City, CA, USA). The actin gene (GenBank: AK458277.1) was used as the reference gene. The PCR reaction and program were modified according to the

Peer) reviewing PDF | (2020:09:52609:3:0:NEW 21 Dec 2020) 
manual. The PCR reaction (a total reaction volume of $10 \mu \mathrm{L}$ ) comprised $5 \mu \mathrm{L} 2 \times \mathrm{SYBR}$ Green PCR Master Mix, $3 \mu \mathrm{L}$ of the cDNA product, $1 \mu \mathrm{L}$ of primer mix, and $1 \mu \mathrm{L}$ of DNase/RNasefree water. The quantitative PCR thermal cycler program included $95^{\circ} \mathrm{C}$ for $10 \mathrm{~s}$, followed by 40 cycles at $95^{\circ} \mathrm{C}$ for $5 \mathrm{~s}$ and $60^{\circ} \mathrm{C}$ for $31 \mathrm{~s}$. All primers for q-PCR were synthesized by the same company (AoKe, yangling) (Table S4).

\section{RESULTS}

\subsection{Identification of the SNARE protein in wheat}

To identify SNARE proteins in wheat, the HMMER profile was implemented to identify the wheat genomes. The results showed that 173 hypothetical TaSNARE genes were characterized from wheat databases (Table S1). Qa, Qb, Qc, Qb+Qc and R SNARE proteins comprised $48(27.7 \%), 37(21.4 \%), 39(22.5 \%), 13(7.5 \%)$ and 36(20.8\%) respectively. Among all 21 subfamilies, SYP1 contained a maximum of 33 proteins, and VAMP72 had the second most, at 15 proteins (Table S1). The encoded proteins comprised between 121 and 466 amino acid residues, the PIs ranged from 4.72 to 9.65 , and the molecular weights were distributed from 13687.37 to 51665.97 Da (Table S1).

All the sequences were divided into 64 groups in wheat (Table S1). Among these groups, 38 groups representing 114 genes contained three genes from each of the different subgenomes that were regarded as orthologous copies of a single $S N A R E$ gene named triplet. Five groups contained different homoeologous genes that were from the same homoeologous group (e.g., TaSYP43-4AL, TaSYP43-7AS, and TaSYP43-7DS). Eight groups contained two genes (e.g., TaSYP131-2BS and TaSYP131-2DS), and the remaining 8 groups consisted of only one gene (e.g., TaGOS12-6BS). Five groups had four genes, among which 4 groups had tandemly repeated genes (e.g., TaSNAP1-2A1, TaSNAP1-2A2,TaSNAP1-2B, and TaSNAP1-2D). We found that the Go term "vesicle-mediated transport" was most significantly enriched in the SNARE proteins (Table S2).

3.2 Chromosomal locations and gene collinearity analysis of $S N A R E$ gene family members in wheat

Most collinear gene pairs occur within the same chromosome group (Fig 1). The chromosomal distribution of the SNARE gene family of $T$. aestivum was analyzed. Fig. 2 revealed the chromosomal location of $173 S N A R E$ genes. All 21 wheat chromosomes have several SNARE gene family members: the wheat 1 to 7 homoeologous groups had 14 $(1 \mathrm{~A}=5,1 \mathrm{~B}=4,1 \mathrm{D}=5), 22(2 \mathrm{~A}=7,2 \mathrm{~B}=8,2 \mathrm{D}=7), 31 \quad(3 \mathrm{~A}=12,3 \mathrm{~B}=9,3 \mathrm{D}=10), 25(4 \mathrm{~A}=9,4 \mathrm{~B}=9$ 
212

213

214

215

216

\section{7}

218

219

220

221

222

223

224

225

226

227

228

229

230

231

232

233

234

235

236

237

238

239

240

241

242

243

244

245

246

$4 \mathrm{D}=7), 23(5 \mathrm{~A}=8,5 \mathrm{~B}=9,5 \mathrm{D}=6), 26(6 \mathrm{~A}=9,6 \mathrm{~B}=9,6 \mathrm{D}=8)$ and $27(7 \mathrm{~A}=11,7 \mathrm{~B}=8,7 \mathrm{D}=9)$ SNARE genes, and 4 had no chromosomal location. In addition to homoeologous group 1 , the SNARE genes were evenly distributed in the wheat genome, and the number of genes on each chromosome is similar. The most striking result to emerge from Fig 2 was that the triplets, which were from different subgenomes, were similar in terms of their relative position on chromosomes.

\subsection{Phylogenetic, motif and structural analysis of the $S N A R E$ family genes}

To further analyze the phylogeny, motif and structure of TaSNAREs, we selected one protein (genome group A chromosomal priority selection) from each of the 64 groups and obtained 64 SNAREs. The results showed that these proteins were primarily divided into 5 clades (Fig 3). Most clades had three homoeologous proteins in the same branch, and these three homoeologous proteins were from three chromosomes in the same homoeologous group.

It is apparent from Fig 3 that $S N A R E s$ in different subfamilies had different motifs. Qa had motifs 1, 2, 5, 8, 11 and 13. Qb had motifs 6, 7, 10, 12 and 13. Qc had motifs 5, 9 and 13. Qb+Qc had motifs 9 and 12 . R had motifs 3, 4, 13 and 14. The results showed that motifs $6,8,10,11,13$ and 15 were not predicted as being present in these SNAREs. Motif 1 was the SNARE domain; motifs 2 and 5 were syntaxin domains; motif 3 was the synaptobrevin domain, motifs 4 and 14 were longin domains; motif 7 was the SEC20 domain; and motif 12 was the V-SNARE-C domain. Qa, Qb, Qc, and R had motif 13, which was located in the C-terminus and associated SNAREs with lipid bilayers, and this motif was named the transmembrane (TM) domain (Lipka et al., 2007).

\subsection{Cis-acting elements of TaSNARE genes}

Further analysis of the 2000-bp promoter upstream of the 5' end of the TaSNARE gene was performed. This promoter contains 9 types of resistance-related cis-acting elements (Table S3), including W-box (Cis-I), germ-related (Cis-II), MYB (Cis-III), SA-responsive (Cis-IV), Ethresponsive (Cis-V), EIRE (Cis-VI), G-box (Cis-VII), H-box (Cis-VIII) and IAA-responsive (CisIX) elements.

As shown in Fig 4, Cis-I to Cis-IX were represented by 2230, 5054, 1647, 882, 170, 225, 152, 118 and 309 elements, respectively, in all 173 SNARE gene promoters. Cis-I, Cis-II and Cis-III made up $82.92 \%$ of all disease-related elements. Among the Cis-I elements, TaUSE12-7A was the most abundant (36). Among the Cis-II elements, TaSEC222-5B was the most abundant (87). Among the Cis-III elements, TaSEC222-5A was the most abundant (19).

In one triplet, for the promoters of the resistance-related elements, the numbers were similar. However, there were exceptions, as follows: TaSFT11-2A/B/D had 5/21/16 Cis-I elements; TaNPSN12-4A/B/D had 71/17/21 Cis-II elements, and TaSYP222-6A/B/D had 3/11/1 Cis-IV elements. 


\subsection{Expression analysis of TaSNARE genes by RNA-seq}

To further understand the functions of the SNARE genes, we extracted gene expression information for 54 genes from six published RNA-seq databases (Fig 5).

As shown in Fig 5, in the growth period of wheat, the SNARE gene is expressed in roots, stems, leaves, seeds and spikes, showing low levels in seeds and leaves and high levels in roots, stems, and spikes. In the seeds of Z75, the expression levels of most genes (45) were very low, and in Z71-Z75-Z85, a high-low-high expression pattern was observed. Many genes (36) were most highly expressed in the 20 DPA aleurone layer during seed development. Most SNARE genes (49) are better expressed under light conditions than under dark conditions. Among these genes, SYP122-6A showed higher expression under light than in the dark in DV92, but in G3116, the opposite trend was observed. Compared with the control, the expression of 22 genes was upregulated $6 \mathrm{~h}$ after stress (drought $6 \mathrm{~h}$, heat $6 \mathrm{~h}$ or drought plus heat $6 \mathrm{~h}$ ), and the expression patterns of 9 genes showed the opposite trend. In the process of leaf senescence, 42 genes showed the highest expression at 12 DAA. More than half of the genes (33) had the following expression distribution pattern in the grain layers: outer pericarp $>$ inner pericarp $>$ endosperm. In the powdery mildew pathogen stress, 3 genes were down-regulated by more than 0.5 times in 24 hours, and one gene was up-regulated by more than 1 times. In total, 17 genes were upregulated by more than 0.5 times, of which 8 genes were up-regulated by more than double. There were 6 genes down-regulated by more than 0.5 times at $48 \mathrm{~h}$, and one gene was downregulated by more than 1 times. There were 12 genes up-regulated by more than 0.5 times, of which 5 genes were up-regulated by more than 1 time. At $72 \mathrm{~h}, 5$ genes were down-regulated by 0.5 times, of which 2 genes were down-regulated by more than 1 time. There were 13 genes upregulated by more than 0.5 times. There were 6 genes that were continuously up-regulated between $24 \mathrm{~h}-72 \mathrm{~h}$. TaSYP135 continued to be down-regulated between $24 \mathrm{~h}-72 \mathrm{~h}$. These genes with the most dramatic changes in expression may have played a role in responding to powdery mildew infection.

\subsection{Expression patterns of TaSNARE genes under powdery mildew treatment}

We selected one gene from each of the 21 classes, and we obtained 21 TaSNARE genes (TaYKT6 had no signal) specific to the designed primers (Table S3). As shown in Fig 6, the expression patterns of different SNARE genes in the same sample and subfamily were similar. Most of the TaSNARE genes had similar expression pattern in 7M US and CS, but in 7M CH, a different expression pattern was observed. A majority of the TaSNARE genes in $7 \mathrm{M} \mathrm{CH}$ had high expression levels at 6h. TaSYP4, TaSYP8, TaMEMB and TaSEC22 had high expression levels at $6 \mathrm{~h}$ in 'Shaanyou 225' but not in the other wheat.

In the Qa subfamily, the expression of all genes changed little at each time point in CS. SYP121, SYP221, and SYP3 were upregulated in the 7M $\mathrm{CH}$ 6h sample but downregulated in 'Shaanyou 225'. SYP4 and SYP8 were upregulated in the 'Shaanyou 225' 6h sample but not in 
284 the 7M CH sample. QaSNARE expression was similar between 7M US and 'Shaanyou 225'. This 285 may indicate that the up-regulated expression of SYP4 and TaSYP8 played a negative role in the 286 wheat response to powdery mildew infection.

In the $\mathrm{Qb}$ subfamily, GOS12 expression was upregulated at $6 \mathrm{~h}$ and then downregulated in four wheat varieties. $M E M B$ expression was upregulated in 'Shaanyou 225' at $6 \mathrm{~h}, 24 \mathrm{~h}$, and $48 \mathrm{~h}$ but not in CS. There was no significant difference in the expression at different time points in $7 \mathrm{M} \mathrm{CH}$ and 7M US. The VTI12 expression patterns were similar to those of MEMB in 'Shaanyou 225' and 7M CH. NPSN11 expression in four wheat varieties were similar, showing downregulation at $6 \mathrm{~h}-48 \mathrm{~h}$, except for the upregulation at $24 \mathrm{~h}-48 \mathrm{~h}$ observed in $7 \mathrm{M} \mathrm{CH}$. SEC203 was downregulated at $6 \mathrm{~h}-48 \mathrm{~h}$ in $7 \mathrm{M}$ US and CS. This gene was downregulated at $12 \mathrm{~h}-$ $24 \mathrm{~h}$ and upregulated at $48 \mathrm{~h}$ in 'Shaanyou 225'. In 7M CH, SEC203 was downregulated at 24h, and the other genes were upregulated. This shows that the NPSN11 gene has little effect in the early stage of wheat's response to powdery mildew infection, while GOS12 and SEC203 play a certain role.

In the Qc subfamily, all QcSNAREs in the same variety were similar. In CS and 7M US, most genes were downregulated at $6-24 \mathrm{~h}$. In $7 \mathrm{M} \mathrm{CH}$, certain genes were downregulated at $24 \mathrm{~h}$, while the others were upregulated. In 'Shaanyou 225', the genes were downregulated at $12 \mathrm{~h}-24 \mathrm{~h}$ and upregulated at $6 \mathrm{~h}$ and $48 \mathrm{~h}$. This means that after powdery mildew infects wheat for $48 \mathrm{~h}$, BET1, SFT1, USE12, and SYP5 were resistant to 7M CH up-regulation and susceptible to 7M US down-regulation, which implies that the up-regulation of these genes has a positive effect in these two types of wheat.

In the $\mathrm{Qb}+\mathrm{Qc}$ subfamily, $S N A P 1$ was upregulated at $6 \mathrm{~h}$ in all varieties and downregulated at 12h-48h, except in 'Shaanyou 225' at 48h, in which SNAPl was upregulated. This may indicate that the $S N A P 1$ gene may play a similar role in the four types of wheat.

In the R subfamily, in CS, VAMP712 was upregulated at $6 \mathrm{~h}$ and at the other time points, it showed no change. In 'Shaanyou 225', VAMP712 was downregulated at $6 \mathrm{~h}$ and $24 \mathrm{~h}$, and at the other time points there was no change. In $7 \mathrm{M} \mathrm{CH}, V A M P 712$ was upregulated at $6 \mathrm{~h}, 12 \mathrm{~h}$, and $48 \mathrm{~h}$ and showed no change at 24h. In 7M US, VAMP712 was upregulated at $24 \mathrm{~h}$ and downregulated at the other time points. No signal for VAMP723 was detected in 'Shaanyou 225'. In $7 \mathrm{M}$ US, VAMP723 was upregulated at $24 \mathrm{~h}$ and downregulated at other time points. In $7 \mathrm{M}$ $\mathrm{CH}, V A M P 723$ was upregulated at $12 \mathrm{~h}$ and downregulated at $24 \mathrm{~h}$ and $48 \mathrm{~h}$. In CS, VAMP723 was upregulated at $6 \mathrm{~h}$ and downregulated at $48 \mathrm{~h}$. SEC222 was upregulated at every time point in 'Shaanyou 225', and the other varieties showed no significant difference. This implies that the up-regulated expression of SEC222 plays a negative role in the powdery mildew infection of 'Shaanyou 225'.

With the evolution of plants, wheat SNARE genes are constantly changing. SNARE genes have different expression patterns after being infected by powdery mildew, suggesting that these genes may be involved in the biological stress response to powdery mildew in different aspects. 


\section{DISCUSSION}

SNAREs are mainly involved in membrane-related life activities. It is apparent from previous reports that $S N A R E S$ are rarely described from the perspective of gene families.

The completion of the wheat genome sequencing work will help in the analysis of key genes and agronomic traits of wheat at the genomic level. However, the sequencing work has progressed slowly because of the very large size of the genome and the high number of repeated sequences. In this paper, 173 nonredundant SNARE genes were obtained from the newly published IWGSC 1.0 wheat genome reference sequence. In another study, using more stringent identification methods, 27 SNARE and 8 NPSN genes were discovered(Gaggar et al. 2020). Common wheat is a heterogeneous hexaploid crop, and it usually contains three paralogous homoeologous genes from groups $\mathrm{A}, \mathrm{B}$, and $\mathrm{D}$, which can be called a triplet. However, in our study, 16 out of 64 groups did not appear as triplets (Table S1). This could be explained by the loss of these genes during long-term evolution, or it could be due to insufficient sequencing depth or incomplete splicing. There are also some triplets in which $\mathrm{A} / \mathrm{B} / \mathrm{U}$ occurs, possibly because the difficulty in splicing leads to the genes not being appropriately located in their chromosomes.

As evidenced by the analysis of cis-acting elements, genes in the same triad are most alike in their components. However, there remain a few differences, which may lead to some bias in the expression of these homoeologous genes in some physiological states. In addition, depending on the composition of cis-acting elements found, the genes mainly contained W-boxes, diseaserelated elements and MYBs. This suggested that SNARE resistance in plants may mainly be regulated by transcription factors such as WRKY, MYB and other disease resistance genes.

Some interesting information was obtained by analyzing the RNA-seq data. Because these RNA-seq databases are relatively old, the genetic information used was a genetic sketch of wheat. We compared the TGAC v1.0 data to a sketch database to find the corresponding SNARE gene in the sketch. The expression patterns of triplet genes in the same group were very similar, and in photomorphogenesis, most of the triplets did not show gene expression data for the B and D genomes, so we selected the A genome in the triplet. The gene was analyzed, and if there was no group A gene, a gene of group B or D was used.

Members of the same class as the subfamilies have diverse roles in the same life activities. In Arabidopsis, severe male gametophytic defects occur only when syp123, syp125, and syp 131 were simultaneously mutated (Slane et al. 2017). Arabidopsis SCYL2B and CHC1 undergo vesicle transport through VTI11 or VTI12 for plant growth (Jung et al. 2017). On the other hand, homoeologous genes may also play different roles. Overexpression of OsVAMP7111 did not enhance rice resistance to blast, while overexpression of OSVAMP714 increased the resistance. This suggests that VAMP714 is potentially specific for resistance to rice blast (Sugano et al., 2016). PEN1 in plants forms the SNARE complex with VAMP721 and VAMP722 during defense against powdery mildew fungi, and it also forms SNARE complexes in vitro with VAMP724 and VAMP727, which are not related to plant immunity (Kwon et al. 2008). PVA31 
361 participates in SA-associated apoptosis by interacting with $V A M P 721 / 722 / 724$ but not 362 VAMP711/727 to combat pathogen infection (Ichikawa et al. 2015). In wheat, silencing 363 of TaNPSN11/13 reduced the resistance to CYR23, whereas silencing of TaNPSN12 did not have the same effect (Wang et al. 2014).

The RNA-seq data showed that homoeologous genes in the same evolutionary branch exhibit many different expression patterns under the same conditions, as observed for $V T I 11 / 12 / 13 / 14$ and GOS11/12, but there were some differences. For example, NPSN11/13 were in one class, and NPSN12 was not clustered with NPSN11/13. In the senescing leaf time course, NPSN11 and NPSN13 exhibited

low-high-low expression patterns, while NPSN12 showed no difference in expression at each stage. During photomorphogenesis, the expression of NPSN12 and NPSN13 in the dark was higher than that in the light, and that of NPSN11 showed no difference. In the heat and drought treatments, both NPSN12/13 were downregulated compared to the control, and there was no change in expression from 1 to $6 \mathrm{~h}$ after treatment. On the other hand, after treatment, NPSN11 was upregulated under drought, upregulated at high temperature and upregulated $1 \mathrm{~h}-6 \mathrm{~h}$ after treatment. In another group of subfamily genes, the $S N A P$ genes, the expression patterns differed greatly among the three members. The expression level of $S N A P 3$ (FPKM) was higher than that of $S N A P 1 / 4$ in each period and process. In the developmental time course of wheat, SNAP3 was the most highly upregulated in all tissues at various developmental stages, while $S N A P 4$ was downregulated, and $S N A P 1$ expression was low. In the grain layers, the expression of $S N A P 1$ had the distribution endosperm $>$ outer pericarp>inner pericarp, SNAP2 expression had the distribution endosperm=inner pericarp $>$ outer pericarp, and $S N A P 4$ expression had the distribution outer pericarp>inner pericarp >endosperm. Under heat and drought and in the senescing leaf time course, only SNAP3 expression was high, while the other FPKM values were less than 1. After powdery mildew infection, the RNA-seq results were different from the quantitative results. This could be explained from the following aspects. First, there was a big difference between the materials of this study and Zhang et al., 2014. The N9134 material was the offspring of tetraploid durum wheat, and this study, CS additional lines were formed by crossing with Ae. geniculata. Second, in the time of expression change, most of the resistant materials that we quantified reached their peak at $6 \mathrm{~h}$, while RNA-seq reached the peak at $24 \mathrm{~h}$. The expression in $24 \mathrm{~h}$ was not clear. However, the expression level of this family gene will be changed by the signals from powdery mildew infection, suggesting that SNARE plays a certain role.

$\mathrm{CS}$ is the parent of $\mathrm{A}$ and $\mathrm{B}$, and the other parents are different varieties of Ae. geniculata. However, their resistances to powdery mildew are quite different. We chose these two materials to try to explain the effects of exogenous chromosomes on endogenous gene expression from a genomic perspective. Fig 6 shows that the 7M US and CS expression patterns were similar after infection with powdery mildew, but the expression pattern of $7 \mathrm{M} \mathrm{CH}$ was very different from those of the other two varieties. This suggests that our exogenous chromosomes had some effect on the endogenous gene expression and may have led to differences in resistance. It has been reported that after the introduction of exogenous chromosomes, genes on exogenous $7 \mathrm{M}^{\mathrm{g}}$ chromosomes mainly affect homologous genes on homologous chromosomes. The resistance 
402

403

gene carried on $7 \mathrm{M}^{\mathrm{g}}$ may affect the expression of $S N A R E$-related genes. Therefore, we propose two hypotheses. The first is that the resistance gene of exogenous $7 \mathrm{M}^{\mathrm{g}}$ could resist powdery mildew by participating in the disease resistance pathway of wheat. Second, it is possible that the exogenous $7 \mathrm{M}^{\mathrm{g}}$ chromosome achieves resistance to powdery mildew by affecting the expression of the endogenous seventh homologous gene.

In conclusion, this paper identified 173 SNAREs in wheat, which laid a foundation for further studies on the function of SNARE genes. In addition, these results will also be helpful for further study of the powdery mildew resistance of wheat.

\section{References}

Assaad FF, Qiu J-L, Youngs H, Ehrhardt D, Zimmerli L, Kalde M, Wanner G, Peck SC, Edwards H, Ramonell K, Somerville CR, and Thordal-Christensen H. 2004. The PEN1 syntaxin defines a novel cellular compartment upon fungal attack and is required for the timely assembly of papillae. Molecular biology of the cell 15:51185129. 10.1091/mbc.e04-02-0140

Bock JB, Matern HT, Peden AA, and Scheller RH. 2001. A genomic perspective on membrane compartment organization. Nature 409:839-841. 10.1038/35057024

Bracuto V, Appiano M, Zheng Z, Wolters AA, Yan Z, Ricciardi L, Visser RGF, Pavan S, and Bai Y. 2017. Functional Characterization of a Syntaxin Involved in Tomato (Solanum lycopersicum) Resistance against Powdery Mildew. Front Plant Sci 8:1573. 10.3389/fpls.2017.01573

Choulet F, Alberti A, Theil S, Glover N, Barbe V, Daron J, Pingault L, Sourdille P, Couloux A, Paux E, Leroy P, Mangenot S, Guilhot N, Le Gouis J, Balfourier F, Alaux M, Jamilloux V, Poulain J, Durand C, Bellec A, Gaspin C, Safar J, Dolezel J, Rogers J, Vandepoele K, Aury JM, Mayer K, Berges H, Quesneville H, Wincker P, and Feuillet C. 2014. Structural and functional partitioning of bread wheat chromosome 3B. Science 345:1249721. 10.1126/science.1249721

Chung KP, Zeng Y, Li Y, Ji C, Xia Y, and Jiang L. 2018. Signal motif-dependent ER export of the Qc-SNARE BET12 interacts with MEMB12 and affects PR1 trafficking in Arabidopsis. J Cell Sci 131:jcs202838. 10.1242/jcs.202838

Collins NC, Thordal-Christensen H, Lipka V, Bau S, Kombrink E, Qiu JL, Hückelhoven R, Stein M, Freialdenhoven A, Somerville SC, and Schulze-Lefert P. 2003. SNAREprotein-mediated disease resistance at the plant cell wall. Nature 425:973-977. 10.1038/nature02076

Douchkov D, Nowara D, Zierold U, and Schweizer P. 2005. A high-throughput genesilencing system for the functional assessment of defense-related genes in barley epidermal cells. Mol Plant Microbe Interact 18:755-761. 10.1094/mpmi-18-0755

Eschen-Lippold L, Landgraf R, Smolka U, Schulze S, Heilmann M, Heilmann I, Hause G, and Rosahl S. 2012. Activation of defense against Phytophthora infestans in potato 
440

441

442

443

444

445

446

447

448

449

450

451

452

453

454

455

456

457

458

459

460

461

462

463

464

465

466

467

468

469

470

471

472

473

474

475

476

477

478

479

480

by down-regulation of syntaxin gene expression. New Phytol 193:985-996. 10.1111/j.1469-8137.2011.04024.x

Fox SE, Geniza M, Hanumappa M, Naithani S, Sullivan C, Preece J, Tiwari VK, Elser J, Leonard JM, Sage A, Gresham C, Kerhornou A, Bolser D, McCarthy F, Kersey P, Lazo GR, and Jaiswal P. 2014. De novo transcriptome assembly and analyses of gene expression during photomorphogenesis in diploid wheat Triticum monococcum. PLoS One 9:e96855. 10.1371/journal.pone.0096855

Gaggar P, Kumar M, and Mukhopadhyay K. 2020. Genome-Scale identification, in silico characterization and interaction study between Wheat SNARE and NPSN gene families involved in Vesicular Transport. IEEE/ACM Trans Comput Biol Bioinform Pp. 10.1109/tcbb.2020.2981896

He X, Huo Y, Liu X, Zhou Q, Feng S, Shen X, Li B, Wu S, and Chen X. 2018. Activation of disease resistance against Botryosphaeria dothidea by downregulating the expression of MdSYP121 in apple. Hortic Res 5:24. 10.1038/s41438-018-0030-5

Ichikawa M, Nakai Y, Arima K, Nishiyama S, Hirano T, and Sato MH. 2015. A VAMPassociated protein, PVA31 is involved in leaf senescence in Arabidopsis. Plant Signal Behav 10:e990847. 10.4161/15592324.2014.990847

Jones DT, Taylor WR, and Thornton JM. 1992. The rapid generation of mutation data matrices from protein sequences. Comput Appl Biosci 8:275-282. 10.1093/bioinformatics/8.3.275

Jung J-Y, Lee DW, Ryu SB, Hwang I, and Schachtman DP. 2017. SCYL2 Genes Are Involved in Clathrin-Mediated Vesicle Trafficking and Essential for Plant Growth. Plant Physiol 175:194. 10.1104/pp.17.00824

Kalde M, Nühse TS, Findlay K, and Peck SC. 2007. The syntaxin SYP132 contributes to plant resistance against bacteria and secretion of pathogenesis-related protein 1. Proc Natl Acad Sci U S A 104:11850-11855. 10.1073/pnas.0701083104

Karnik R, Grefen C, Bayne R, Honsbein A, Köhler T, Kioumourtzoglou D, Williams M, Bryant NJ, and Blatt MR. 2013. Arabidopsis Sec1/Munc18 protein SEC11 is a competitive and dynamic modulator of SNARE binding and SYP121-dependent vesicle traffic. Plant Cell 25:1368-1382. 10.1105/tpc.112.108506

Kumar S, Stecher G, and Tamura K. 2016. MEGA7: Molecular Evolutionary Genetics Analysis Version 7.0 for Bigger Datasets. Mol Biol Evol 33:1870-1874. 10.1093/molbev/msw054

Kwon C, Neu C, Pajonk S, Yun HS, Lipka U, Humphry M, Bau S, Straus M, Kwaaitaal M, Rampelt H, El Kasmi F, Jürgens G, Parker J, Panstruga R, Lipka V, and SchulzeLefert P. 2008. Co-option of a default secretory pathway for plant immune responses. Nature 451:835-840. 10.1038/nature06545

Larkin MA, Blackshields G, Brown NP, Chenna R, McGettigan PA, McWilliam H, Valentin F, Wallace IM, Wilm A, Lopez R, Thompson JD, Gibson TJ, and Higgins DG. 2007. Clustal W and Clustal X version 2.0. Bioinformatics 23:2947-2948. 10.1093/bioinformatics/btm404 
481

482

483

484

485

486

487

488

489

490

491

492

493

494

495

496

497

498

499

500

501

502

503

504

505

506

507

508

509

510

511

512

513

514

515

516

517

518

519

520

521
Lipka U, Fuchs R, and Lipka V. 2008. Arabidopsis non-host resistance to powdery mildews. Curr Opin Plant Biol 11:404-411. 10.1016/j.pbi.2008.04.004

Lipka V, Kwon C, and Panstruga R. 2007. SNARE-ware: the role of SNARE-domain proteins in plant biology. Annu Rev Cell Dev Biol 23:147-174. 10.1146/annurev.cellbio.23.090506.123529

Liu Z, Xin M, Qin J, Peng H, Ni Z, Yao Y, and Sun Q. 2015. Temporal transcriptome profiling reveals expression partitioning of homeologous genes contributing to heat and drought acclimation in wheat (Triticum aestivum L.). BMC Plant Biol 15:152. 10.1186/s12870-015-0511-8

Ma J, Chen J, Wang M, Ren Y, Wang S, Lei C, Cheng Z, and Sodmergen. 2018. Disruption of $O S S E C 3 A$ increases the content of salicylic acid and induces plant defense responses in rice. J Exp Bot 69:1051-1064. 10.1093/jxb/erx458

Nielsen ME, Feechan A, Böhlenius H, Ueda T, and Thordal-Christensen H. 2012. Arabidopsis ARF-GTP exchange factor, GNOM, mediates transport required for innate immunity and focal accumulation of syntaxin PEN1. Proc Natl Acad Sci U S A 109:11443-11448. 10.1073/pnas.1117596109

Pearce S, Huttly AK, Prosser IM, Li YD, Vaughan SP, Gallova B, Patil A, Coghill JA, Dubcovsky J, Hedden P, and Phillips AL. 2015. Heterologous expression and transcript analysis of gibberellin biosynthetic genes of grasses reveals novel functionality in the GA3ox family. BMC Plant Biol 15:130. 10.1186/s12870-0150520-7

Pearce S, Tabbita F, Cantu D, Buffalo V, Avni R, Vazquez-Gross H, Zhao R, Conley CJ, Distelfeld A, and Dubcovksy J. 2014. Regulation of $\mathrm{Zn}$ and Fe transporters by the GPCl gene during early wheat monocarpic senescence. BMC Plant Biol 14:368. 10.1186/s12870-014-0368-2

Pfeifer M, Kugler KG, Sandve SR, Zhan B, Rudi H, Hvidsten TR, Mayer KF, and Olsen OA. 2014. Genome interplay in the grain transcriptome of hexaploid bread wheat. Science 345:1250091. 10.1126/science.1250091

Söllner T, Whiteheart SW, Brunner M, Erdjument-Bromage H, Geromanos S, Tempst P, and Rothman JE. 1993. SNAP receptors implicated in vesicle targeting and fusion. Nature 362:318-324. 10.1038/362318a0

Sanderfoot A. 2007. Increases in the number of SNARE genes parallels the rise of multicellularity among the green plants. Plant Physiol 144:6-17. 10.1104/pp.106.092973

Schilde C, Lutter K, Kissmehl R, and Plattner H. 2008. Molecular identification of a SNAP-25-like SNARE protein in Paramecium. Eukaryot Cell 7:1387-1402. 10.1128/ec.00012-08

Slane D, Reichardt I, El Kasmi F, Bayer M, and Jürgens G. 2017. Evolutionarily diverse SYP1 Qa-SNAREs jointly sustain pollen tube growth in Arabidopsis. Plant $J$ 92:375-385. 10.1111/tpj.13659

Snyder BA, and Nicholson RL. 1990. Synthesis of phytoalexins in sorghum as a site- 
specific response to fungal ingress. Science 248:1637-1639. 10.1126/science.248.4963.1637

Sugano S, Hayashi N, Kawagoe Y, Mochizuki S, Inoue H, Mori M, Nishizawa Y, Jiang CJ, Matsui M, and Takatsuji H. 2016. Rice OsVAMP714, a membrane-trafficking protein localized to the chloroplast and vacuolar membrane, is involved in resistance to rice blast disease. Plant Mol Biol 91:81-95. 10.1007/s11103-016-0444-0

Sun Q, Wang G, Zhang X, Zhang X, Qiao P, Long L, Yuan Y, and Cai Y. 2017. Genome-wide identification of the TIFY gene family in three cultivated Gossypium species and the expression of JAZ genes. Sci Rep 7:42418. 10.1038/srep42418

Uemura T, Kim H, Saito C, Ebine K, Ueda T, Schulze-Lefert P, and Nakano A. 2012. Qa-SNAREs localized to the trans-Golgi network regulate multiple transport pathways and extracellular disease resistance in plants. Proc Natl Acad Sci USA 109:1784-1789. 10.1073/pnas.1115146109

Walther-Larsen H, Brandt J, Collinge DB, and Thordal-Christensen H. 1993. A pathogen-induced gene of barley encodes a HSP90 homologue showing striking similarity to vertebrate forms resident in the endoplasmic reticulum. Plant Mol Biol 21:1097-1108. 10.1007/bf00023606

Wang D, and Dong X. 2011. A highway for war and peace: the secretory pathway in plant-microbe interactions. Mol Plant 4:581-587. 10.1093/mp/ssr053

Wang X, Wang X, Deng L, Chang H, Dubcovsky J, Feng H, Han Q, Huang L, and Kang Z. 2014. Wheat TaNPSN SNARE homologues are involved in vesicle-mediated resistance to stripe rust (Puccinia striiformis f. sp. tritici). J Exp Bot 65:4807-4820. 10.1093/jxb/eru241

Wang Y, Long D, Wang Y, Wang C, Liu X, Zhang H, Tian Z, Chen C, and Ji W. 2020. Characterization and Evaluation of Resistance to Powdery Mildew of WheatAegilops geniculata Roth 7M (7A) Alien Disomic Substitution Line W16998. Int J Mol Sci 21. 10.3390/ijms21051861

Wang Y, Quan W, Peng N, Wang C, Yang X, Liu X, Zhang H, Chen C, and Ji W. 2016. Molecular cytogenetic identification of a wheat-Aegilops geniculata Roth $7 \mathrm{M}^{\mathrm{g}}$ disomic addition line with powdery mildew resistance. Molecular Breeding 36:40. 10.1007/s11032-016-0463-1

Wick P, Gansel X, Oulevey C, Page V, Studer I, Dürst M, and Sticher L. 2003. The expression of the t-SNARE AtSNAP33 is induced by pathogens and mechanical stimulation. Plant Physiol 132:343-351. 10.1104/pp.102.012633

Zadoks JC, Chang TT, and Konzak CF. 1974. A decimal code for the growth stages of cereals. Weed Research 14:415-421. https://doi.org/10.1111/j.13653180.1974.tb01084.x

Zhang H, Yang YZ, Wang CY, Liu M, Li H, Fu Y, Wang YJ, Nie YB, Liu XL, and Ji WQ. 2014. Large-scale transcriptome comparison reveals distinct gene activations in wheat responding to stripe rust and powdery mildew. BMC genomics 15. Artn $89810.1186 / 1471-2164-15-898$ 
Zhang X, Zhao H, Gao S, Wang WC, Katiyar-Agarwal S, Huang HD, Raikhel N, and Jin H. 2011. Arabidopsis Argonaute 2 regulates innate immunity via miRNA393(*)mediated silencing of a Golgi-localized SNARE gene, MEMB12. Mol Cell 42:356366. 10.1016/j.molcel.2011.04.010 
Figure 1

SNARE collinear gene pairs in wheat

The innermost line represents a collinear gene pair, in which the SNARES gene pair are coloured and the others are gray. The length of the innermost colored arc is the size of the chromosome. Each yellow line in the middle is a gene. The outer red line shows the gene density. 


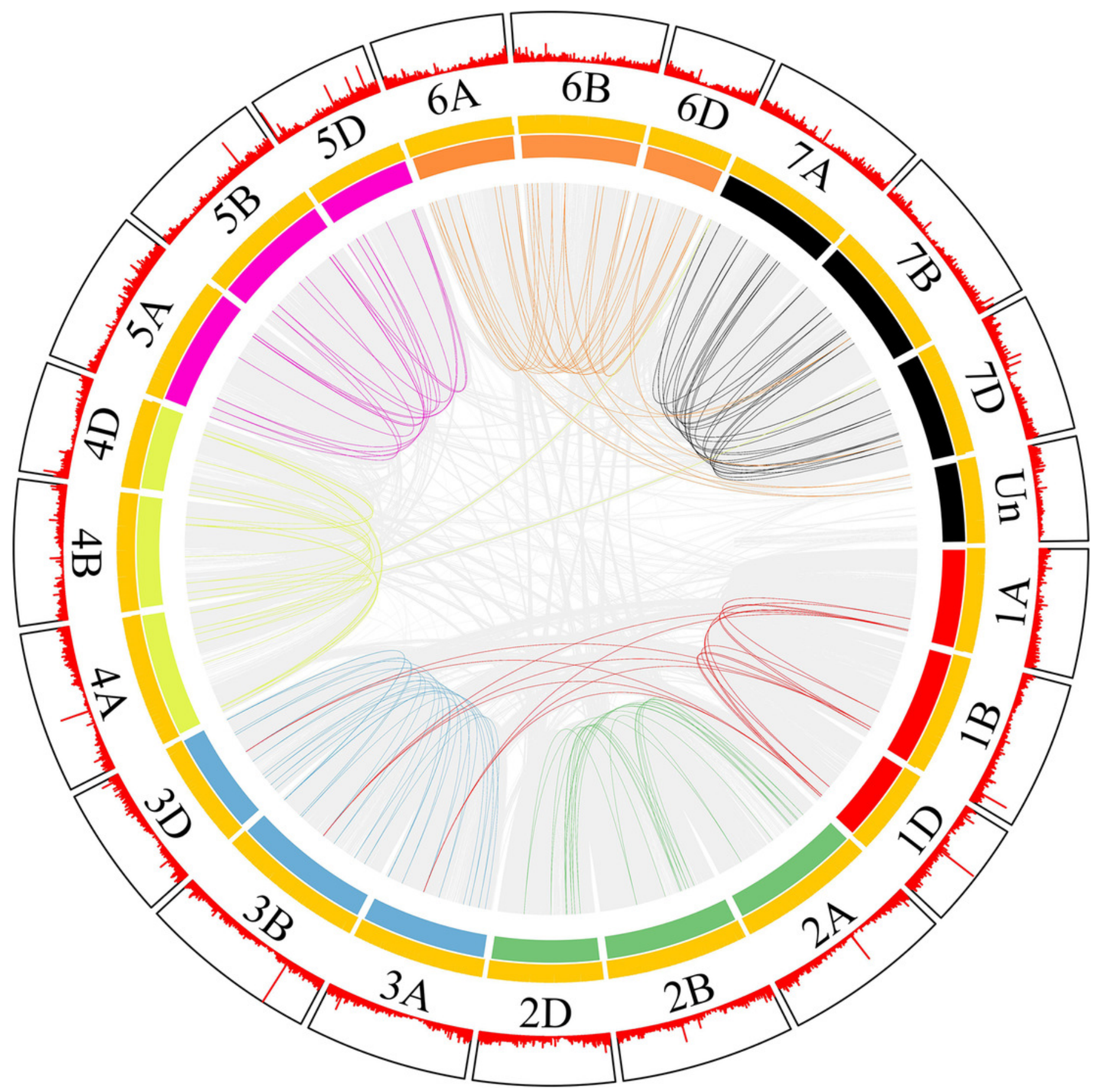


Figure 2

\section{Chromosomal locations of SNARE genes in wheat}

A total of 173 SNARE genes were localized to Triticum aestivum. Qa SNARE: Green. Qb SNARE: Fuchsia. Qc SNARE: Brown. Qb+Qc SNARE: Red. R SNARE: Blue.
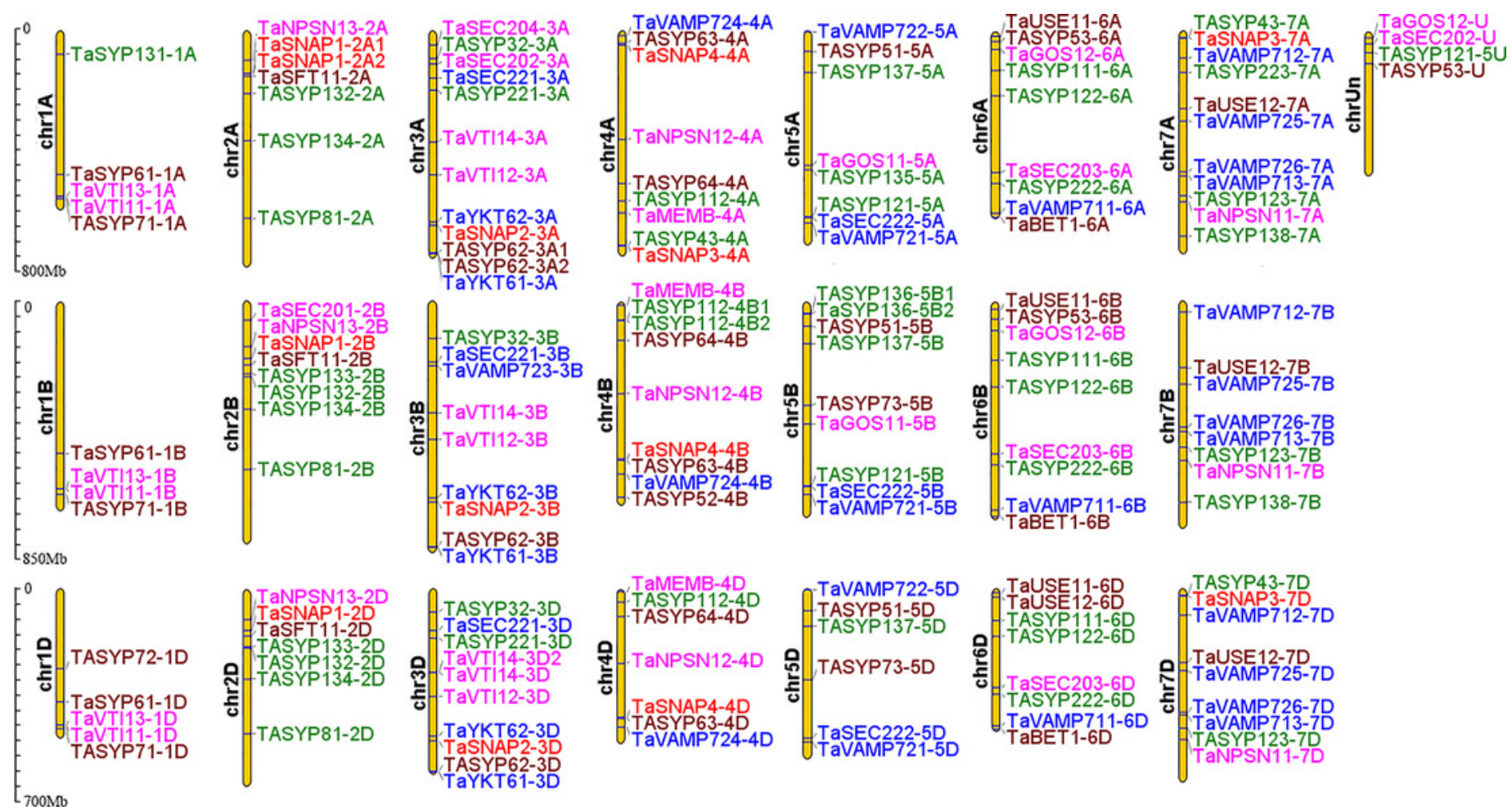
Figure 3

Phylogenetic analysis, gene structure, domain location and motif compositions of the SNARE gene family in wheat
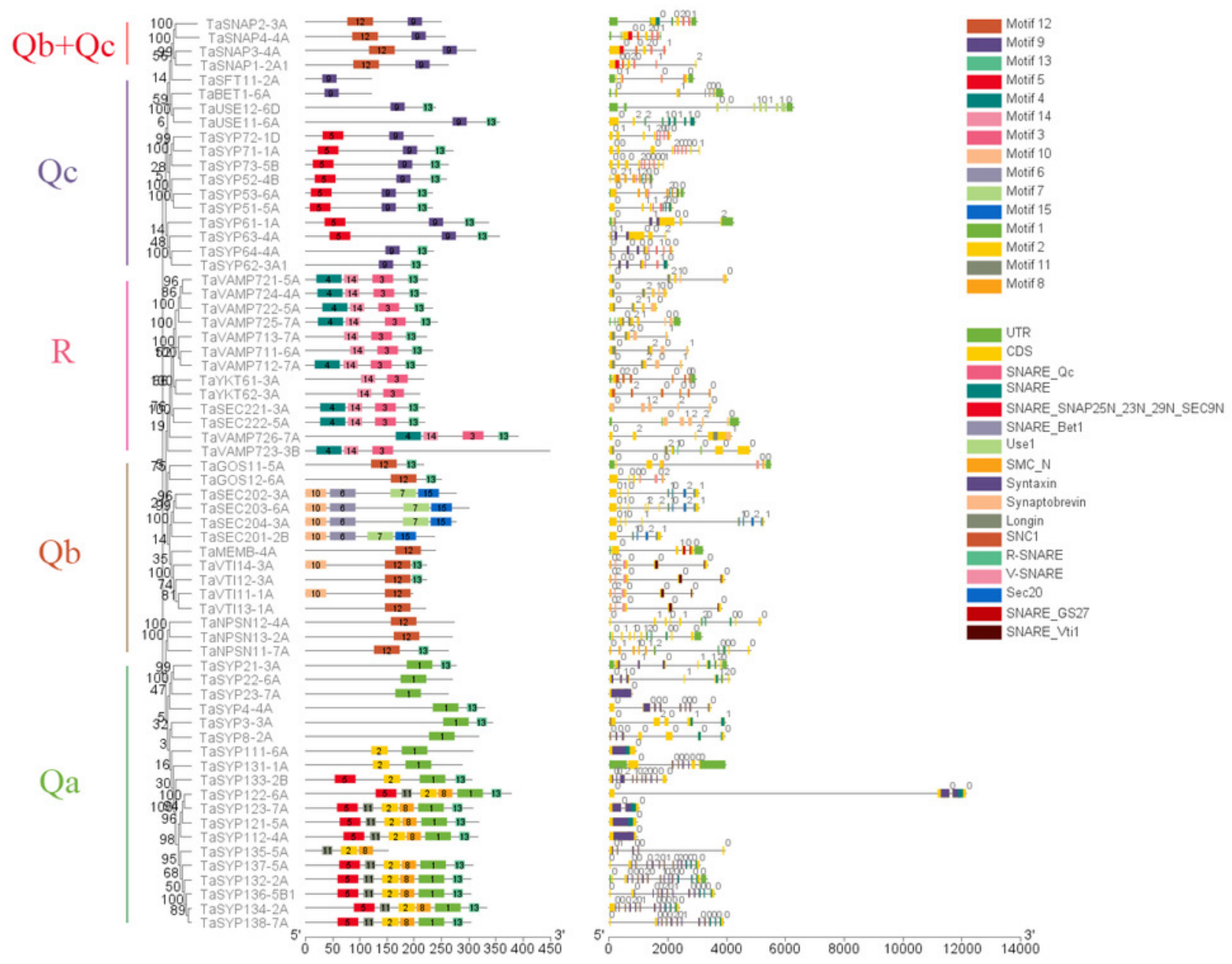


\section{Figure 4}

The number and proportion of 9 disease-related cis-acting element in SNARE genes promoter

Cis-I: W-box; Cis-II:Germs-related; Cis-III:MYB; Cis-IV:SA responsible; Cis-V:Eth responsible; Cis-VI:EIRE; Cis-VII:G-box; Cis-VIII:H-box; Cis-IX:IAA responsible.

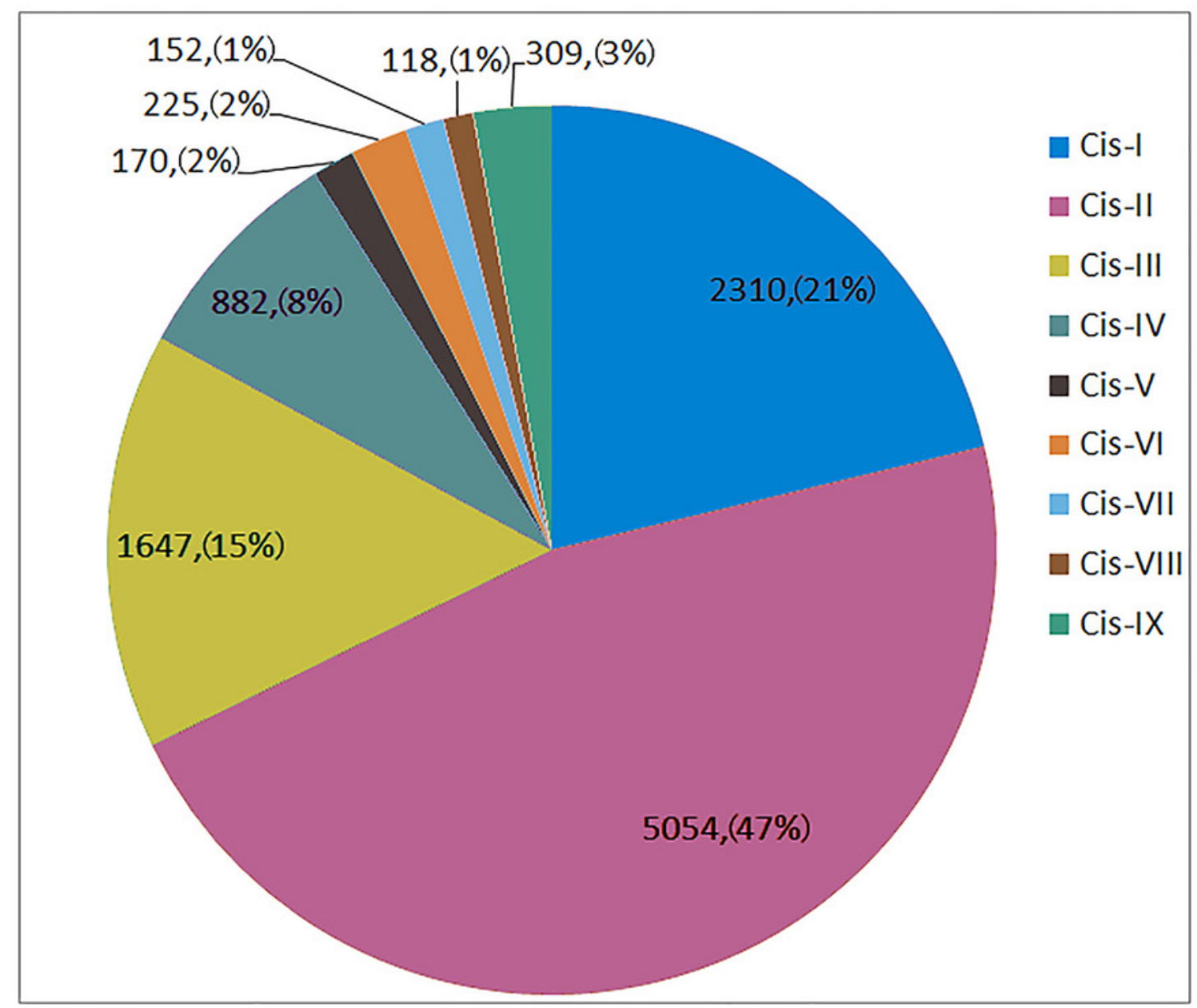




\section{Figure 5}

The expression profiles of TaSNARE genes in deferent treatment and stage

Developmental time course: Z10-Z85. Grain layers at 12 DPA: outer pericarp, inner pericarp and endosperm. Grain layer developmental time course: 10 DPA whole endosperm, 20 DPA whole endosperm, 20 DPA starchy endosperm, 20 DPA transfer cells, 20 DPA aleurone, 30 DPA starchy endosperm, 30 DPA aleurone plus endosperm. Senescing leaf time course: HD, 12 DAA and 22 DAA. Photomorphogenesis for DV92 and G3116. Drought and heat: control, drought $1 \mathrm{~h}$, drought $6 \mathrm{~h}$, heat $1 \mathrm{~h}$, heat $6 \mathrm{~h}$, drought plus heat $1 \mathrm{~h}$, drought plus heat $6 \mathrm{~h}$. Powdery mildew pathogen stress: included non-innoculation, powdery24h, powdery $48 \mathrm{~h}$ and powdery $72 \mathrm{~h}$. 


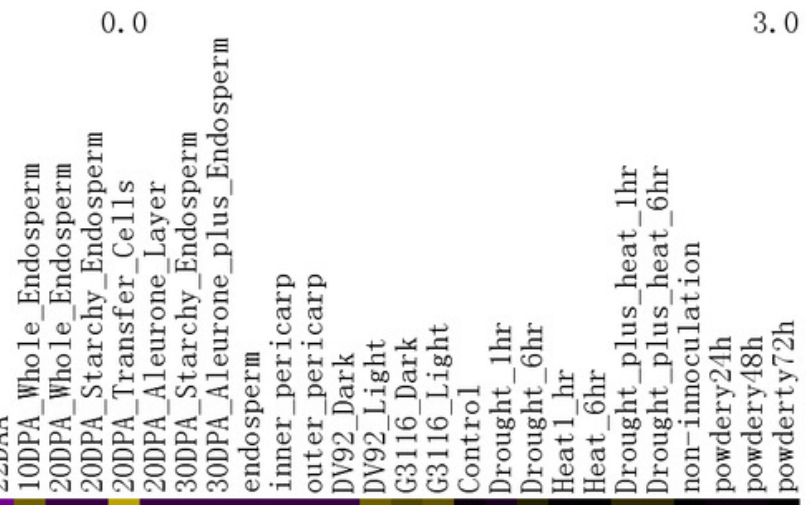

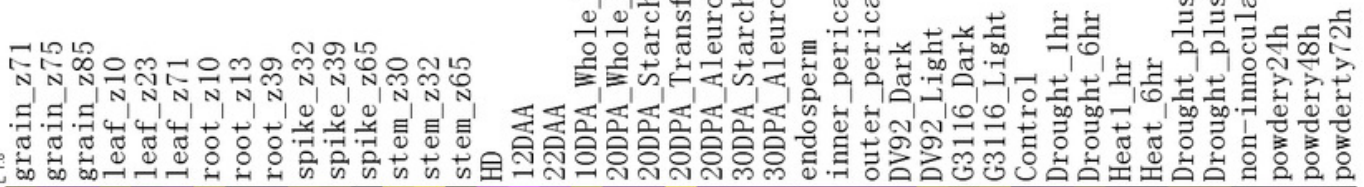

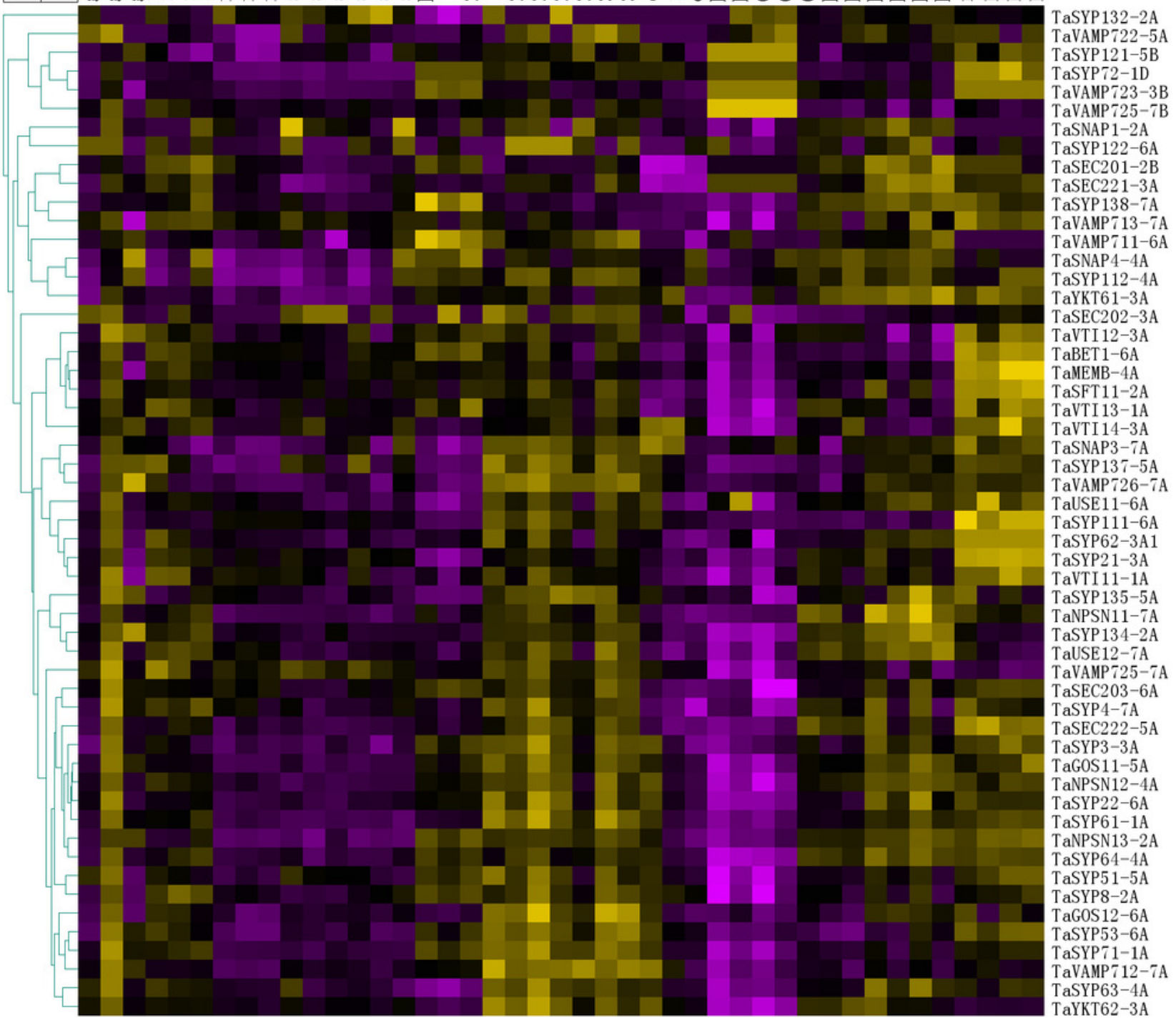


Figure 6

TASNARE genes expression patterns infected by Bgt E09

CS: susceptible, Chinese spring. 7M CH: resistant, NA0973-5-4-1-2-9-1 (CS-SY159 DA 7M"9, (CS)/ Ae. geniculata SY159//CS)). 7M US: susceptible, TA7661 (CS-AEGEN DA 7M).

Shaanyou225: susceptible, wheat cultivate variety Shaanyou225. The 20 parts of Figure 6 should be labeled A-T.
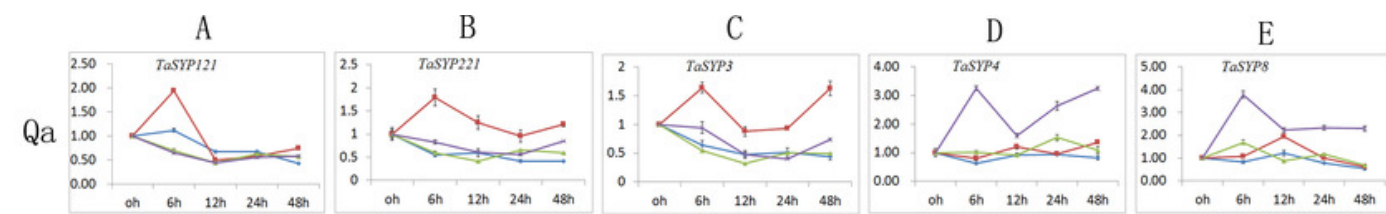

$$
\mathrm{Qb}
$$
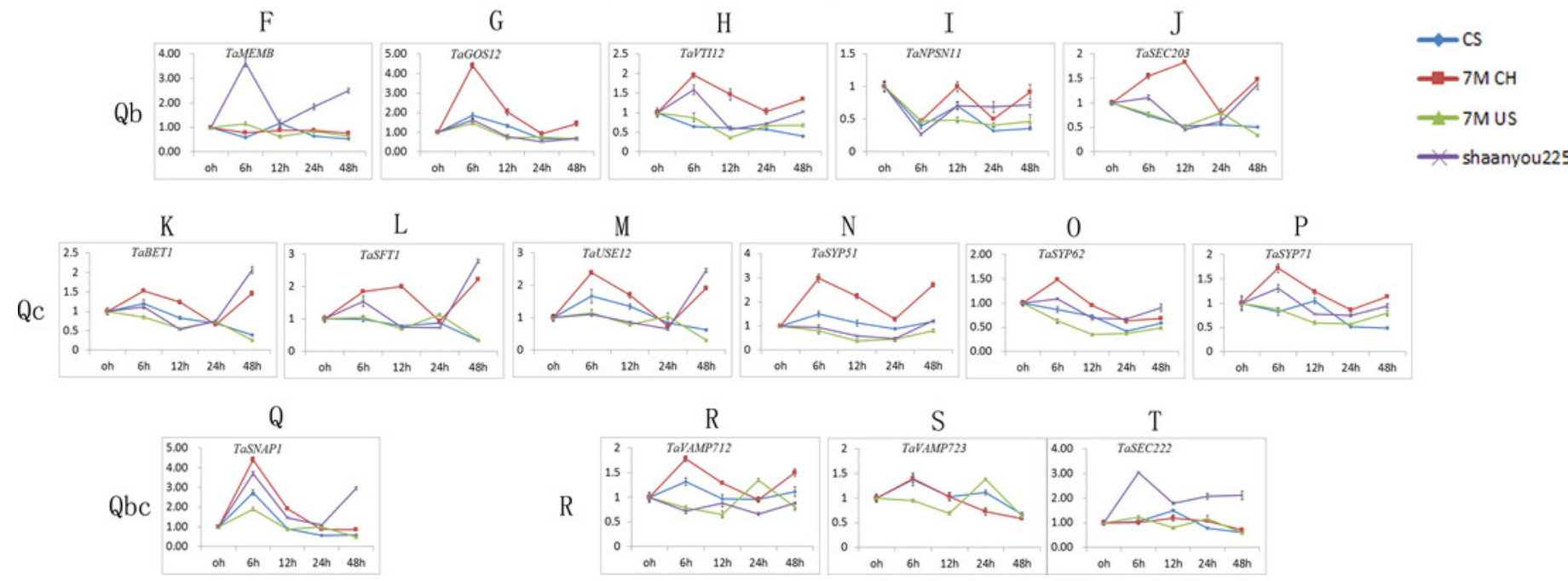